\title{
Application of Electronic Speckle Pattern Interferometry for Evaluation of the Static Stiffness of a Complex Orthopedic Implant
}

\author{
Piotr Mrozek ${ }^{1 *}$, Piotr Borkowski ${ }^{1}$ \\ 1 Institute of Biomedical Engineering, Faculty of Mechanical Engineering, Bialystok University of Technology, \\ Wiejska 45C, 15-351 Bialystok, Poland \\ * Corresponding author's email: p.mrozek@pb.edu.pl
}

\begin{abstract}
The paper presents a comparative analysis of the results of experimental and numerical evaluation of static stiffness of extendable tumor knee joint prosthesis for children. Electronic speckle pattern interferometry and finite element method were used for experimental and computational research, respectively. The procedure of experimental tests shows that the stiffness of the joint prosthesis can be determined experimentally by means of electronic speckle pattern interferometry, but the quantitative result of such a procedure cannot be captured directly in a finite element method model. The values of static stiffness calculated numerically do not fall within the range of experimental results. The discrepancy grows as the extension of the endoprosthesis increases and the value of relative difference between numerical and experimental results reaches the value of $54 \%$. The inconsistency of results can be explained by the involvement of the joint linking the body of the implant to the moving part. While effective in the case of traditional, one-piece constructions, finite element method modeling does not yield satisfactory results in the case of such an elementary parameter as is the static stiffness of the implant, due to the difficulty in estimating the stiffness of the joint present in the sliding node. The use of the experimental method makes it possible to estimate the degree of this inconsistency.
\end{abstract}

Keywords: complex orthopedic implant, electronic speckle pattern interferometry, finite element method

\section{INTRODUCTION}

In biomechanical analysis of implants, there are two significant properties that describe their mechanical characteristics: stiffness and permanent deformation induced by the working load [1]. The stiffness of implants and tissue-implant interfaces is of great significance in analysis of systems of this type. The term "stiffness" describes the relationship between the load applied and the elastic strain corresponding to it. The stiffness of a system depends on the mechanical properties of the materials used to build it as well as on the geometric characteristics of the tested system. Stiffness is typically determined within the linear range of the stress-strain curve. Sometimes, in practice, it is more important to determine the absolute value of displacement under load than the relative value of the system's strain. Hence, the property of stiffness is associated with the absolute change of a part's dimensions and describes the value of load required for an elastic change of a part's dimension by one unit of length. As mentioned above, the second of the mechanical characteristics typically tested is the value of the system's permanent deformation. The permanent deformation value describes the level of damage to a part as a result of applied, increasing working load. There is extensive literature concerning analysis of these two aspects of implants' biomechanical characteristics. Intramedullary implants (tibia - implant system) [1,2], dental bridges affixed to mandibular implants [3, 4], hip joint implants $[5,6]$, stabilizing plates [7-11], vertebral body implants $[12,13]$, SOP (String of Pearls) [14], porous titanium implants [15], humeral heads [16-18] and other implants have been analyzed experimentally and numerically. The 
subject matter of these publications pertains to one-piece implants and their fixation to bone tissue as well as to more complex systems with a geometry that is unchanging throughout the course of their exploitation. The Finite Element Method (FEM) is a tool that is commonly used for analysis. Modeling results remain in good consistency with experimental results obtained by various testing techniques for the systems listed above.

In recent years, implants in the form of a more complex structure have been proposed, essentially being mechanisms with a specific kinematic function. An example of such a structure is, e.g. an endoprosthesis extended in a non-invasive manner [19]. At this time, there are no analyses in the literature that account for the stiffness of implants in the work characteristics of such structures under working load. It could be expected that the mechanical characteristic of such a system will not be constant, but rather dependent on the actual kinematic configuration. It also seems that a description of the system that accounts for the stiffness of the joints between the structure's subassemblies rather than the stiffness of the subassemblies themselves will be more appropriate in this case. Such a description is applied in analysis of machine designs, and in the literature, has not yet been used to describe the characteristics of orthopedic implants.

By employing terminology used to describe machine constructions, it can be stated that the dependency between forces and deformations and their joint influence on the functional properties of a complex implant structure leads to requirements concerning the stiffness of individual parts of the structure and stiffness of the structure as a whole. In practice, the relationship between force $F$ and displacement $\delta$, at a specific point in the structure, is predominantly linear within the entire range of interest of the occurring forces [20]. Static stiffness $k$ is expressed as

$$
\mathrm{k}=\mathrm{F} / \delta
$$

When the directions of $F$ and $\delta$ coincide, the relationship between force and displacement is defined as "direct stiffness" [20]. When a single force is applied to a point of a structure and displacement is measured in an absolute manner - in the coordinate system relating to the entire structure - the relationship between force and absolute displacement is defined as "absolute stiffness". If oppositely directed forces are applied between two points of a structure, the dependency between force and relative displacement of the points is defined as "relative stiffness" [20].

It should be mentioned that there is a strict dependency between the static stiffness and dynamic stiffness of a structure, which seems particularly significant in the case of implants with elongated shapes, and because of this, implants are especially susceptible to vibrations over the course of their use. From a dynamic perspective, the fundamental modes of the structure's free vibrations are of greater significance than higher modes. If damping is distributed regularly in structural components, the fundamental shapes of dynamic strain curves are approximately the same as the shapes of static strain curves [20]. A high ratio of static stiffness to the structure's mass increases the frequency values of its free vibrations. The structure may then liberate itself from any resonance vibrations within the range of the relatively low frequencies of the present working load.

For the above reasons, knowledge of the absolute static stiffness of an implant construction plays a significant role during both the designing stage and operating stage of a system. When a structure's components have relatively simple shapes, the stiffness of a specific part of the structure and of the system as a whole can be calculated by means of strength-of-materials analytical formulas [20]. In the case of more complex shapes, computer-assisted engineering (CAE) methods, including FEM analysis, perform particularly well. Nevertheless, calculating the shares of various parts of a structure in its total stiffness is difficult due to the inability to fully account for the stiffness of joints between individual components [21]. This is why experimental methods for determining the stiffness of systems are widely used. They make it possible to verify numerical models. Methods based on measurements of point displacements in objects under load are applied for experimental determination of static stiffness $[1,7]$. The static characteristics of an object can also be determined by means of dynamic analysis methods. These are single- or multi-point measuring methods that make it possible to determine dependencies between $F$ and $\delta$ at specific, individual places of the structure. However, from the perspective of a designer or user, knowledge of the continuous field of spatial dependencies between forces and displacements in the chain of the system's joined components would be more beneficial. This would make it possible to determine the role and share of individual parts in the 
structure's total stiffness. This article presents the application of measurements conducted by means of electronic speckle pattern interferometry (ESPI) [22] for the purpose of determining dependencies between applied load and the continuous displacement field of an endoprosthesis subjected to extension. The results of these measurements enable determination of both the static stiffness of the endoprosthesis structure and the role of its individual components in its global stiffness characteristic. There are certain reports in the literature on the application of laser interferometry in the field of testing of orthopedic implants [23], nevertheless, no works relate to the application of ESPI for analysis of the static stiffness of endoprostheses with a complex structure.

In summary, due to the continuous development in the field of complex orthopedic implants, there is a need to compare the results of numerical calculations in terms of stiffness with the experimental results. ESPI method seems to be suitable for carrying out measurements with the high accuracy required to verify the results of numerical calculations.

\section{MATERIALS AND METHODS}

\section{ESPI interferometer}

An ESPI interferometer was developed for research purposes [21]. The schematic diagram of the ESPI interferometer is shown in Figure 1. The system's subassemblies are fixed to a thick, massive aluminum plate so as to minimize the level of vibrations, which affect the accuracy of measurement. The beam of light emitted by DPSS laser source 1 (Spectra-Laser, $50 \mathrm{~mW}, \lambda=532 \mathrm{~nm}$ ) is split by light-splitting plate 2 into two coherent beams. The transmitted beam is reflected by mirrors 3 and 4 onto the lens of microscope objective 5. The reflected beam illuminates surface $S_{\text {o }}$ of the object 6 . The beam reflected by beamsplitter 2 falls onto the lens of microscope objective 7 , and after passing through a pair of polarizers 8 and 9 , illuminates reference surface $\mathrm{S}_{\mathrm{r}}$ of diffuser 10, which is made of matte glass. Speckle patterns are generated by light-diffusing surfaces $\mathrm{S}_{\mathrm{o}}$ and $\mathrm{S}_{\mathrm{r}}$. After passing through light-splitting cube 11 and lens 13 of camera 12, speckle patterns meet and mutually interfere on the image plane of CMOS image sensor 14 (Aptina CMOS MT9P006, optical format $1 / 2.5$ ", number of active pixels 2592 x 1944 , pixel size $2.2 \times 2.2 \mu \mathrm{m}$ ) in the camera. The aperture of the camera's lens is adjusted so as to accommodate the speckle size to the pixel size in the CMOS image sensor. When the diameter of the camera's lens aperture is selected correctly, the mean speckle size is slightly larger than the diagonal of a pixel on the detector. The relative intensities of the two beams are regulated by selecting the reflection/transmission ratio of beamsplitter 2 and setting the angular positions of polarizers 8 and 9 . The camera's exposure time is adjusted so as to obtain an image without saturation of the CMOS image sensor. Bitmap files with 2560x1922 resolution (4.92 MP, 24-bit color) are downloaded by PC 15 using USB3 interface. Out-of- plane displacements of the surface $S_{o}$ are measured using immobile surface $S_{r}$, which serves as the surface of reference. The initial image of the speckle pattern is acquired by the camera when no displacement of surface $S_{0}$ is effected. The computer's software periodically

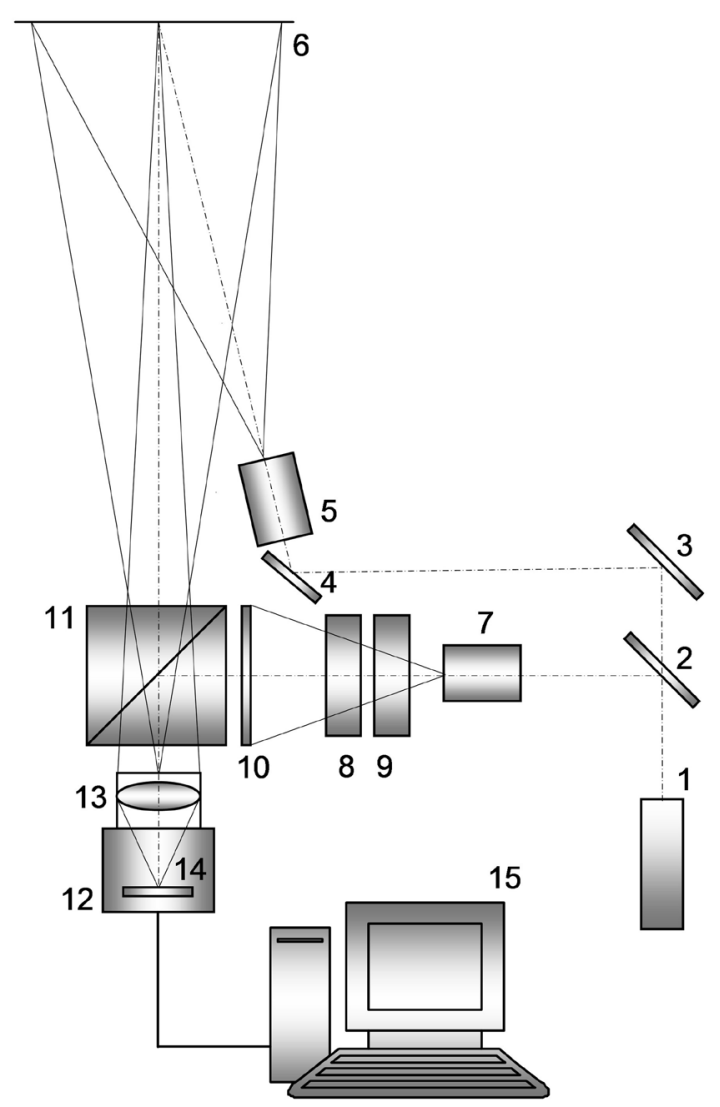

Figure 1. Schematic diagram of the ESPI interferometer's configuration: 1 laser; 2 plate beamsplitter; 3 and 4 mirrors; 5 microscope objective; 6 surface of object $\mathrm{S}_{\mathrm{o}} ; 7$ microscope objective; 8 and 9 polarizers; 10 diffuser made of matte glass with reference surface $\mathrm{S}_{\mathrm{r}}$; 11 cube beamsplitter; 12 digital camera; 13 camera lens; 14 CMOS image sensor; 15 PC 
subtracts the actual speckle pattern image (with deformation of surface $\mathrm{S}_{\mathrm{o}}$ ) from the initial speckle pattern image at a frequency of $10 \mathrm{~Hz}$. The image obtained is displayed on the computer's monitor and saved to the computer's hard disk.

As a result of subtraction of the actual speckle pattern from the initial speckle pattern, a stationary pattern of correlation fringes is obtained for static deformation of the surface. Laser power, detector sensitivity, size of the camera's field of view and the relative intensities of the two measuring beams are selected in a manner that allows for registration of fringe images in high contrast. The dependency between the increment $\Delta$ of the phase difference of the speckle pattern before and after deformation of $\mathrm{S}_{\mathrm{o}}$ and displacement $\mathrm{w}$ of surface $\mathrm{S}_{\mathrm{o}}$ for the type of interferometer presented in Figure 1 can be expressed as:

$$
\Delta=4 \pi \mathrm{w} / \lambda
$$

The phase difference increment corresponding to the centers of two neighboring fringes (of order $i$ and $(i+1)$ ) in the image of correlation fringes is represented by $\Delta=2 \pi$. Using Eq. (2) displacement $\mathrm{w}$ can be expressed as:

$$
w=(\lambda / 2) i
$$

The base measuring sensitivity of the ESPI system, using correlation fringes for out-of-plane measurements, is equal to $\lambda / 2$. For wavelength $\lambda=532 \mathrm{~nm}$ (a green light source of the DPSS laser was used in experiments), the base sensitivity is equal to approx. $260 \mathrm{~nm}$. In the case where it is possible to determine the centers of neighboring fringes: light and dark, the uncertainty of measurement of displacement from the plane can be corrected to a value of half of the base sensitivity, i.e. approx. $130 \mathrm{~nm}$. Details of the verification of the accuracy of ESPI interferometer for the static measurement of out of plane displacement using Renishaw XL-80 measuring system were described in [24]. In brief, a rectangular aluminum plate was clamped at its bottom edge in front of ESPI interferometer. A laser beam reflected from the retroreflector attached at the back side of the plate was used by Renishaw XL-80 measuring system to evaluate the displacement of the retroreflector. Slight bending of the plate resulted in a decrease of a distance to a laser head of XL-80 system and increase of a distance to ESPI interferometer. In this way a measurement of out of plane displacement of a central point of the retroreflector and the corresponding point of surface was possible at the same time using XL-80 system and ESPI interferometer, respectively.

\section{Extendable tumor prosthesis of the knee joint}

Endoprostheses that can be extended in a non-invasive way constitute a special group of products among medical devices. Pursuant to the relevant law, they can be classified into the group of implant products with a measuring function (III) [19]. Their atypical nature, arising from their highly varied structure and measuring functions as well as their relatively narrow area of applications compared to other devices, means that there is no specific standard pertaining exclusively to testing of extendable prostheses - in contrast to the hip and knee joint prostheses commonly used in orthopedics. This is also why, during certification of such products, tests must be presented to demonstrate that the benefit to the patient arising from their application outweighs the risks.

The presented extendable tumor knee joint prosthesis for children (Fig. 2) meets the following requirements [19]:

- replaces the bone fragment removed during surgery,

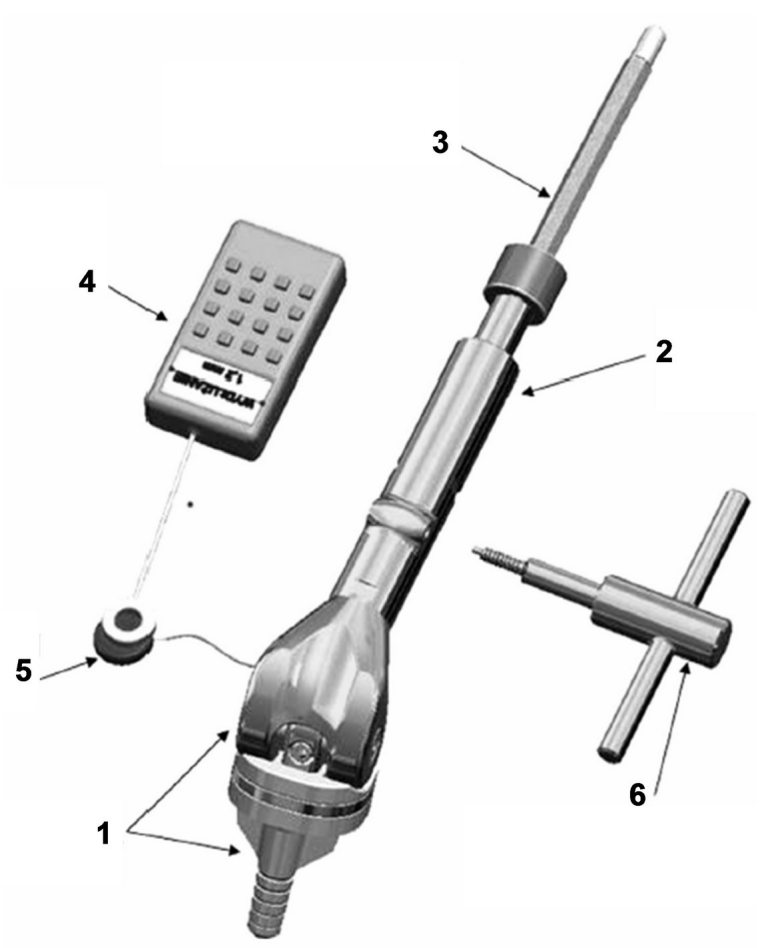

Fig. 2. Extendable endoprosthesis: 1- knee module, 2 - expandable module, 3 - femur stem, 4 - external power and control unit, 5 - internal power and control unit, 6 - emergency elongation system 
- mimics the knee joint's functions, thereby restoring the biomechanics of the skeletal system,

- enables non-invasive extension of the endoprosthesis,

- provides the capability of low-invasive extension of the endoprosthesis.

The extendable endoprosthesis design consists of the following main components:

- knee module,

- extendable module,

- power supply and control system.

The knee module of the endoprosthesis consists of a tibial and femoral component. The extendable module's drive mechanism is situated inside the femoral component and consists of: driveshaft, unidirectional electromagnetic coupling and pinion. The extendable module of the endoprosthesis consists of: exterior sleeve, protrusible sleeve and drive screw. The power supply and control system consists of an external transmission system (outside of the patient's body) and internal reception system (inside of the patient's body along with the endoprosthesis), and it is tasked with controlling the operation of the electromagnetic coupling.

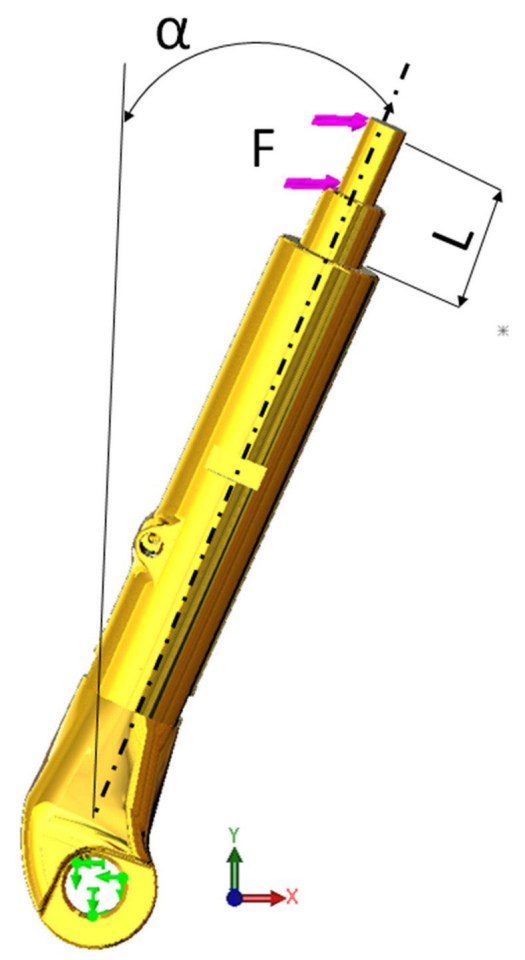

Figure 3. Endoprosthesis model with tibial insert along with boundary conditions

\section{Determination of absolute static stiffness of the endoprosthesis using FEM}

The finite element method (FEM) is currently the basic engineering tool by means of which we can evaluate a designed structure based on a virtual model. FEM modeling was realized taking into consideration the conditions acting on the endoprosthesis over the course of the experiment. The structure of the endoprosthesis was tested in three positions (Fig. 3) of the protrusible sleeve $(\mathrm{L}=15 \mathrm{~mm}, \mathrm{~L}=25 \mathrm{~mm}$ and $\mathrm{L}=55 \mathrm{~mm}$ ). The endoprosthesis axis was deflected from vertical axis $\mathrm{Y}$ by angle $\alpha=22^{\circ}$. Titanium alloy was adopted as the material of the endoprosthesis in simulations (Ti-6Al-4V, Young's modulus E=104.8 GPa, Poisson's ratio $v=0.31$, yield strength $R_{p 0.2}=827$ $\mathrm{MPa}$ ). It was assumed that Ti alloy is isotropic and within a certain load range has linear characteristics. The same load was applied in every case, $\mathrm{F}=0.981 \mathrm{~N}$, applied at the tip of the stem, with a direction conforming to the $\mathrm{X}$ axis. The degrees of freedom corresponding to displacements of the endoprosthesis were substituted by establishing the appropriate constraints in its components. It was assumed that individual components of the structure could interact with one another mutually. At the points of contact between the

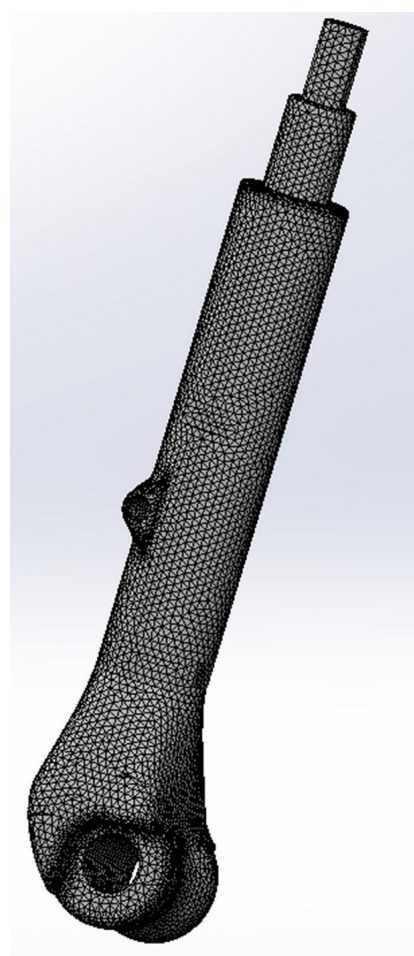

Figure 4. Example of endoprosthesis model discretization by means of finite elements 
module's body and the stem the finite elements had no possibility of interpenetration. All degrees of freedom were removed in the lower part, with displacement support conditions being defined as $\mathrm{u}_{\mathrm{x}}=\mathrm{u}_{\mathrm{y}}=\mathrm{u}_{\mathrm{z}}=0$ in this manner. A spatial, 10-node tetrahedral element was used for analysis. In the numerical modeling, the mesh convergence analysis was taken into account. The calculations were carried out with a gradually increasing number of nodes, while reducing the size of the finite elements. The results obtained in the subsequent stages were compared. For finite element sizes in the range of 2-3 $\mathrm{mm}$, the changes in the obtained results were not significant and such mesh parameters were used for further simulations. The result of division of continuous media into finite elements is shown in Figure 4.

\section{Determination of absolute static stiffness of the endoprosthesis using ESPI}

Experimental tests of mechanical parameters of the endoprosthesis were realized on a laboratory stand equipped with an ESPI laser interferometer, shown in Figure 5. Endoprosthesis 2 , immobilized in a vise fastened to assembly workbench 3, was loaded with force acting in the horizontal direction via tension member 4 , fastened to the tip of the implant's extendable part. Initial load with a value of $1.96 \mathrm{~N}$ was applied, following which the force was increased by the value of the working load, equal to 0.98 $\mathrm{N}$, with simultaneous registration of the image of correlation fringes corresponding to the applied working load.
Geometric configurations of the endoprosthesis, extended by $15 \mathrm{~mm}, 25 \mathrm{~mm}$ and $55 \mathrm{~mm}$, as well as the values and direction of acting load, were adopted according to the parameters applied in numerical simulations, as given in the previous section. A thin strip of white matte film, $0.05 \mathrm{~mm}$ in thickness, was stuck onto the implant's face surface, ensuring correct contrast of interference fringes.

\section{RESULTS}

Figures 6, 7, and 8 present the results of FEM modeling, based on which the absolute static stiffness $\mathrm{k}_{\mathrm{x} \text { FEM }}$ of the endoprosthesis was determined using Eq. (1) for 3 variants:

- extension $15 \mathrm{~mm}, \mathrm{k}_{\mathrm{x} \text { FEM }}=0.98 \mathrm{~N} / 9.091 \cdot 10^{-7} \mathrm{~m}$ $=1.07 \cdot 10^{6} \mathrm{~N} / \mathrm{m}$ (Fig. 6),

- extension $25 \mathrm{~mm}, \mathrm{k}_{\mathrm{x} \text { FEM }}=0.98 \mathrm{~N} / 1.114 \cdot 10^{-6} \mathrm{~m}$ $=0.88 \cdot 10^{6} \mathrm{~N} / \mathrm{m}$ (Fig. 7),

- extension $55 \mathrm{~mm}, \mathrm{k}_{\mathrm{x} \text { FEM }}=0.98 \mathrm{~N} / 2.019 \cdot 10^{-6} \mathrm{~m}$ $=0.47 \cdot 10^{6} \mathrm{~N} / \mathrm{m}$ (Fig. 8$)$.

Figure 9 presents the results of FEM modeling, based on which the absolute static stiffness of the module's body was determined for extension $55 \mathrm{~mm}$, and for displacement of the body edge equal to $826 \mathrm{~nm}$. Experimental results in the form of correlation fringe fields, corresponding to displacements of endoprosthesis components resulting from the applied working load, are presented in Figures 10-12. The displacement $\delta_{\mathrm{x}}$ of endoprosthesis tip was determined taking into account Eq. (3) and the order $\mathrm{n}$ of correlation fringe:

$$
w=(\lambda / 2) i
$$

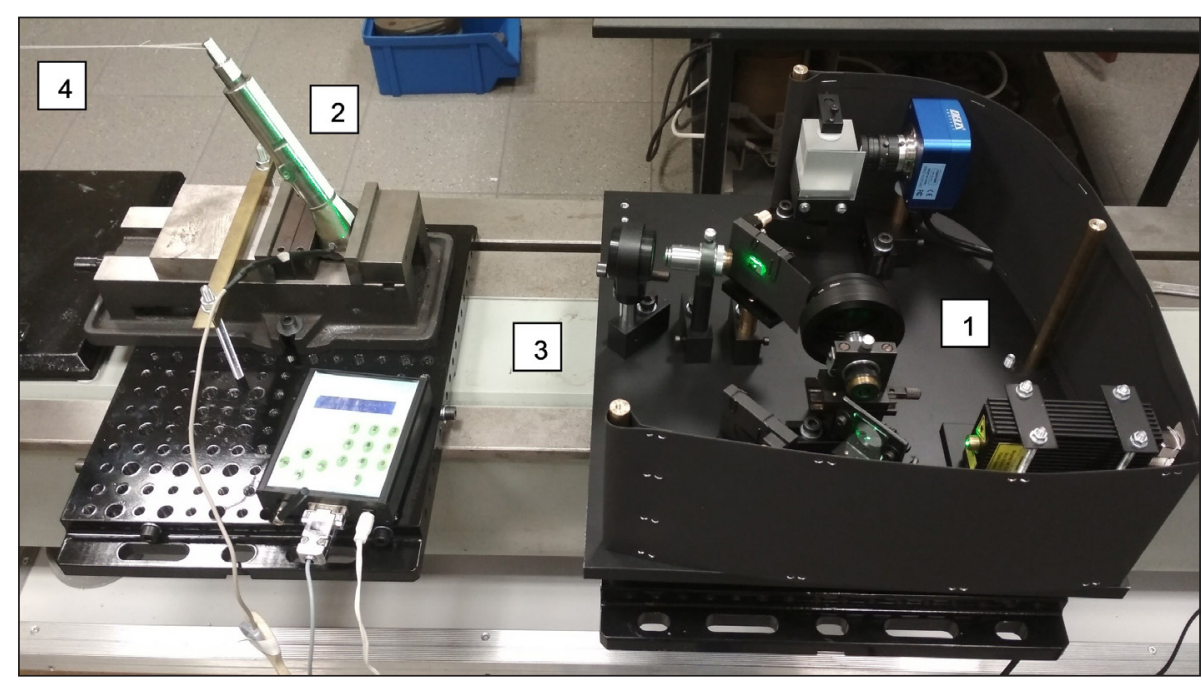

Figure 5. View of ESPI test stand: 1 laser interferometer; 2 endoprosthesis; 3 assembly workbench; 4 tension member carrying working load applied to tip of endoprosthesis 
and the absolute static stiffness $\mathrm{k}_{\mathrm{x}-\mathrm{ESP}}$ of the endoprosthesis was determined for $\lambda / 2=266 \mathrm{~nm}$ using Eq. (4) and Eq. (1) for 3 variants:

- extension $15 \mathrm{~mm}, \mathrm{n}=4.5, \delta_{\mathrm{x} \text { ESPI }}=4.5 \cdot 266$ $\mathrm{nm}=1197 \mathrm{~nm}, \mathrm{k}_{\mathrm{x}_{\mathrm{E} \text { ESPI }}}=0.98 \mathrm{~N} / 1.197 \cdot 10^{-6} \mathrm{~m}=$ $0.82 \cdot 10^{6} \mathrm{~N} / \mathrm{m}$ (Fig. 10),

- extension $25 \mathrm{~mm}, \mathrm{n}=6.5, \delta_{\mathrm{x} \text { ESPI }}=6.5 \cdot 266$ $\mathrm{nm}=1729 \mathrm{~nm}, \mathrm{k}_{\mathrm{x}_{\text {_ESPI }}}=0.98 \mathrm{~N} / 1.729 \cdot 10^{-6} \mathrm{~m}=$ $0.57 \cdot 10^{6} \mathrm{~N} / \mathrm{m}$ (Fig. 11),

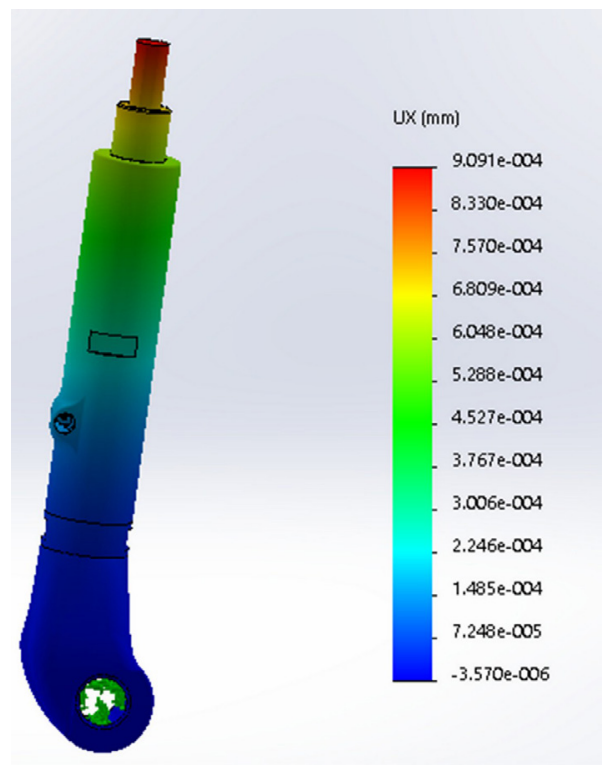

Figure 6. Results of FEM modeling for endoprosthesis extension of $15 \mathrm{~mm}$

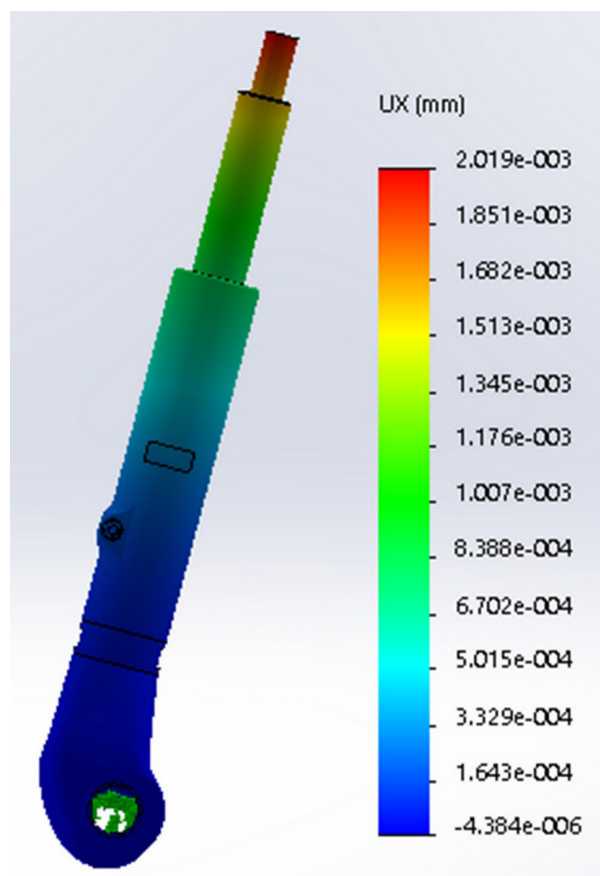

Figure 8. Results of FEM modeling for endoprosthesis extension of $55 \mathrm{~mm}$
- extension $55 \mathrm{~mm}, \mathrm{n}=11.0, \delta_{\mathrm{x}_{\mathrm{ESPI}}}=11.0 \cdot 266$ $\mathrm{nm}=2926 \mathrm{~nm}, \mathrm{k}_{\mathrm{x} \text { ESPI }}=0.98 \mathrm{~N} / 2.926 \cdot 10^{-6} \mathrm{~m}=$ $0.33 \cdot 10^{6} \mathrm{~N} / \mathrm{m}$ (Fig. 12),

- displacement of body edge: $\mathrm{n}=3.5, \delta_{\mathrm{x}_{-} \text {ESPI }}=$ $3.5 \cdot 266 \mathrm{~nm}=931 \mathrm{~nm}$ (Fig. 12).

The results are summarized in Table 1 and Table 2 .

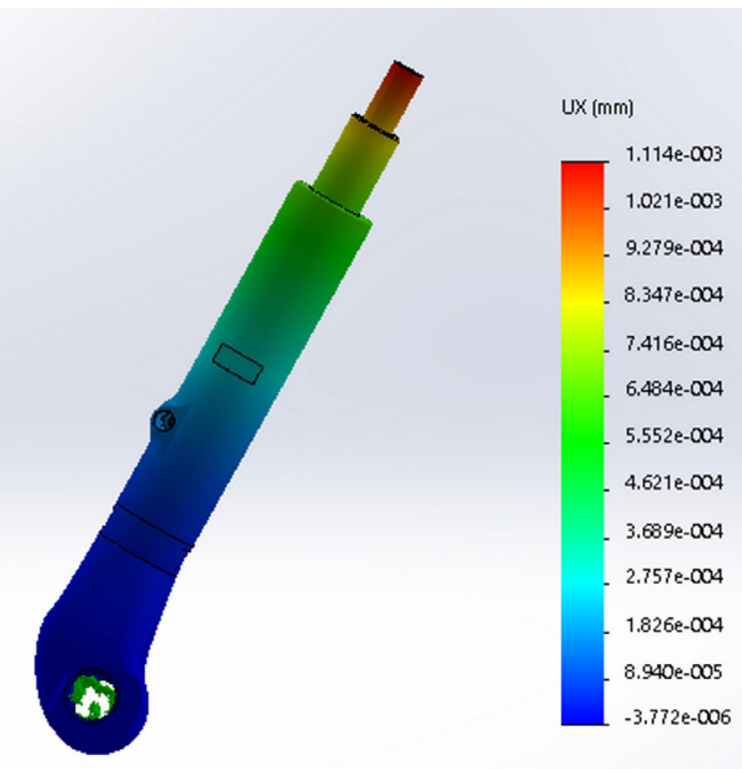

Figure 7. Results of FEM modeling for endoprosthesis extension of $25 \mathrm{~mm}$

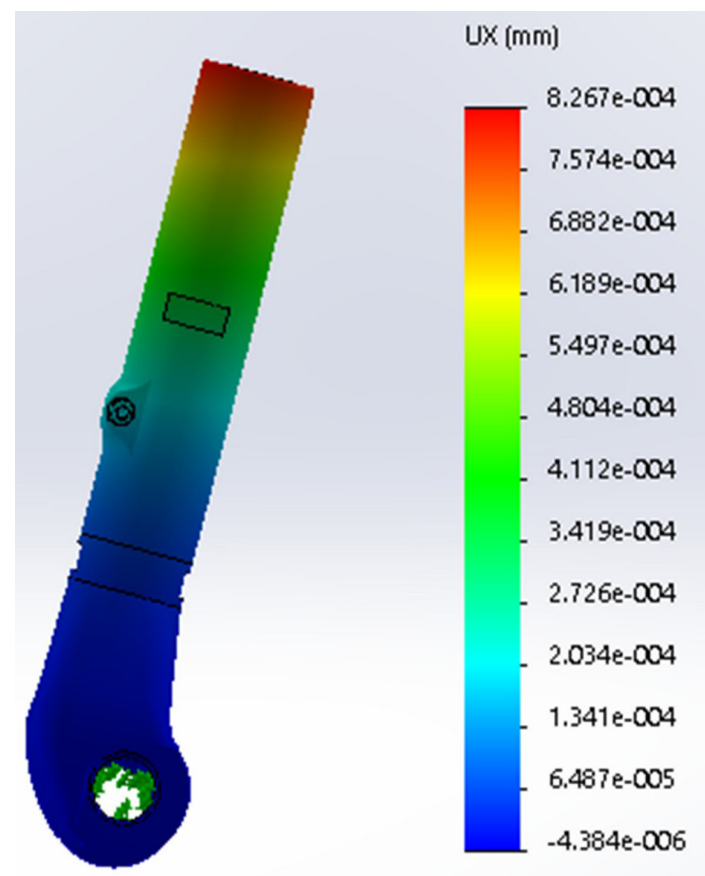

Figure 9. Results of FEM modeling of the endoprosthesis body 

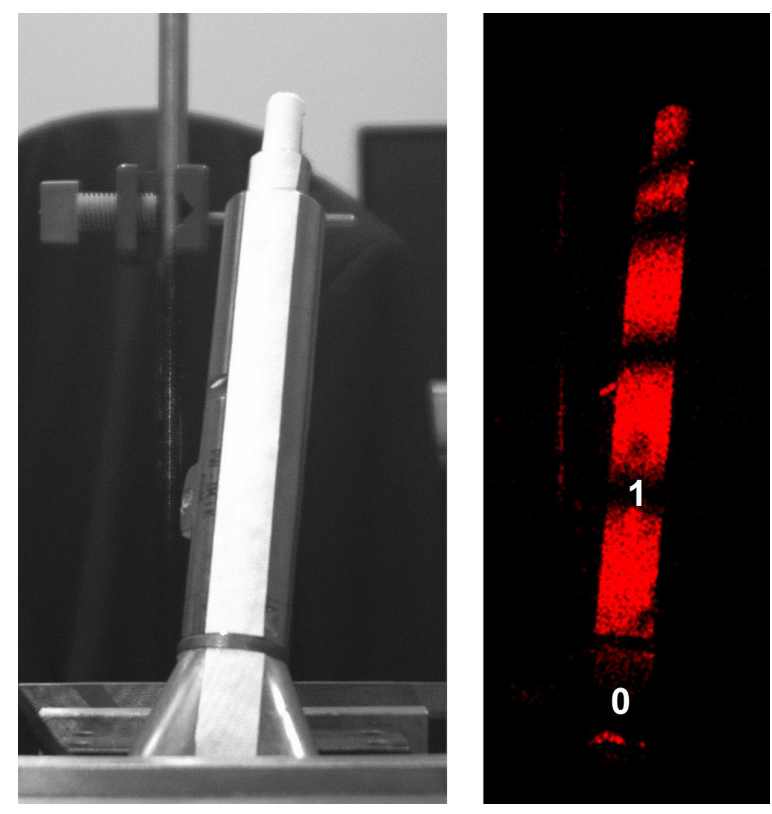

Figure 10. Correlation fringe fields, corresponding to displacements of endoprosthesis components for extension of $15 \mathrm{~mm}$, first two correlation fringes (of zero and 1st order) are indicated
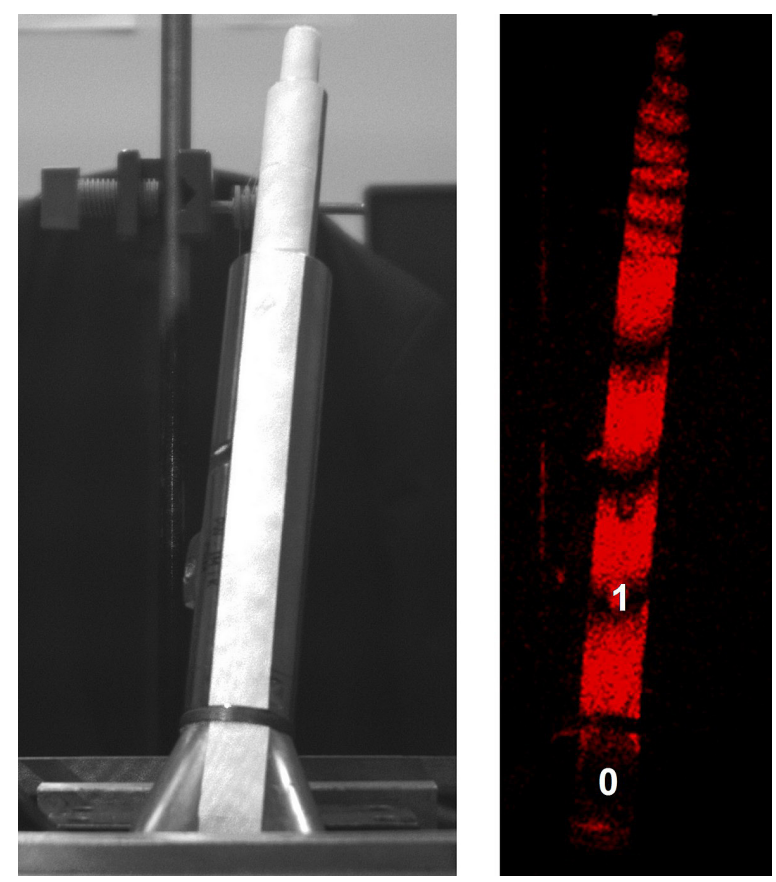

Figure 12. Correlation fringe fields, corresponding to displacements of endoprosthesis components for extension of $55 \mathrm{~mm}$, first two correlation

fringes (of zero and 1st order) are indicated

\section{DISCUSSION}

Based on the data given in Table 1, the results of FEM simulations and ESPI measurements of displacements of the extended module's body
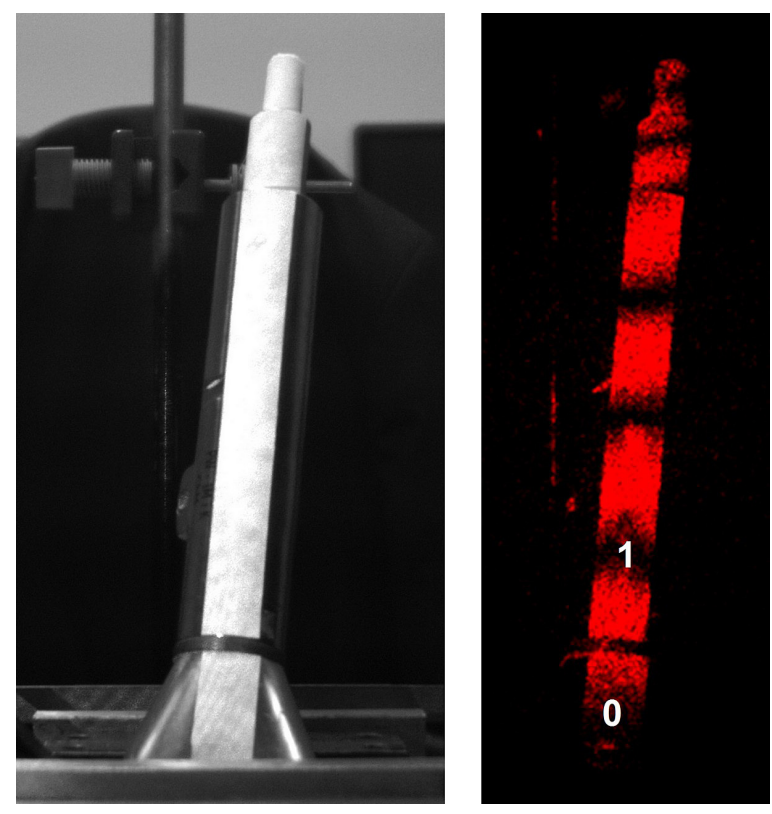

Figure 11. Correlation fringe fields, corresponding to displacements of endoprosthesis components for extension of $25 \mathrm{~mm}$, first two correlation fringes (of zero and 1st order) are indicated

edge for the greatest extension of the endoprosthesis can be compared. FEM and ESPI results were consistent in this scope, considering the range of uncertainty of measurements in the experimental method.

Comparing the results of simulations with experimental values of displacements of the endoprosthesis tip, it can be stated that the values of displacements calculated numerically do not fall within the range of experimental results. The discrepancy grows as the extension of the endoprosthesis increases. The clear inconsistency of results can be explained by the involvement of the joint linking the body of the implant to the moving part. In experimental measurements, this joint's compliance is revealed as it contributes to displacements of the tip under load. No additional elastic deformations occurring at the site of contact between components in the joint node were accounted for in FEM simulations.

The result of not taking into consideration the compliance of the joint's node in numerical simulations as described above shows significant differences in the static stiffness values of the endoprosthesis determined by FEM compared to experimental values. The level of inconsistency, expressed as the relative difference between stiffness values calculated by both methods (Table 2), amounts to $30 \%$ for the smallest extension of the endoprosthesis and has an increasing tendency as extension values increase. 
Table 1. Endoprosthesis body edge and tip displacements determined using FEM and ESPI

\begin{tabular}{|c|c|c|}
\hline $\begin{array}{c}\text { Geometric configuration of } \\
\text { endoprosthesis }\end{array}$ & $\begin{array}{c}\text { Displacement of body edge } \\
\delta_{X_{\text {_FEM }}}, \mathrm{nm}\end{array}$ & $\begin{array}{c}\text { Displacement of body edge } \\
\delta_{\bar{x}_{\text {_ESPI}}}, \mathrm{nm}\end{array}$ \\
\hline \multirow[t]{2}{*}{ extension $55 \mathrm{~mm}$} & 826 & $931+/-130$ \\
\hline & $\begin{array}{c}\text { Displacement of endoprosthesis tip } \\
\delta_{X_{\text {_FEM }}}, \mathrm{nm}\end{array}$ & $\begin{array}{c}\text { Displacement of endoprosthesis tip } \\
\delta_{\text {X_ESPI}_{1}, \mathrm{~nm}}\end{array}$ \\
\hline extension $15 \mathrm{~mm}$ & 909 & $1197+/-130$ \\
\hline extension $25 \mathrm{~mm}$ & 1114 & $1729+/-130$ \\
\hline extension $55 \mathrm{~mm}$ & 2019 & $2926+/-130$ \\
\hline
\end{tabular}

Table 2. Endoprosthesis static stiffness determined using FEM and ESPI

\begin{tabular}{|c|c|c|c|}
\hline $\begin{array}{c}\text { Geometric configuration of } \\
\text { endoprosthesis }\end{array}$ & $\begin{array}{c}\text { Stiffness } \mathrm{k}_{\mathrm{x} F \mathrm{FM}} \text { calculated } \\
\text { based on FEM modeling } \\
\times 10^{6} \mathrm{~N} / \mathrm{m}\end{array}$ & $\begin{array}{c}\text { Stiffness } \mathrm{k}_{\mathrm{x} \text { ESPI }} \text { calculated } \\
\text { based on ESPI measurements } \\
\times 10^{6} \mathrm{~N} / \mathrm{m}\end{array}$ & $\begin{array}{c}\text { Relative difference: } \\
\left(\mathrm{k}_{\mathrm{x}_{-} \mathrm{FEM}}-\mathrm{k}_{\mathrm{x}_{-} \text {ESP }}\right) / \mathrm{k}_{\mathrm{x}_{-} \text {ESPI }}, \%\end{array}$ \\
\hline extension $15 \mathrm{~mm}$ & 1.07 & 0.82 & 30 \\
\hline extension $25 \mathrm{~mm}$ & 0.88 & 0.57 & 54 \\
\hline extension $55 \mathrm{~mm}$ & 0.47 & 0.33 & 42 \\
\hline
\end{tabular}

\section{CONCLUSIONS}

The results of realized tests point to the high level of difficulty in determining basic mechanical characteristics of implants with a complex structure, exemplified here by the extendable endoprosthesis, by means of numerical methods. While effective in the case of traditional, one-piece constructions, FEM modeling does not yield satisfactory results in the case of such an elementary parameter as is the static stiffness of the implant, due to the difficulty in estimating the stiffness of the joint present in the sliding node.

Comparing the values of the endoprosthesis body edge displacement determined using FEM and ESPI methods (Table 1), a good agreement of the numerical and experimental results can be seen. The numerical value of the displacement determined numerically is in the measurement uncertainty field of ESPI method. Thus, the method of numerical calculations used guarantees the feasibility of the results which are characterized by high accuracy in the area covering the dominant part of the analyzed structure. This accuracy is determined by the correct analysis of the mesh convergence (correct element size), appropriately selected material parameters and the type of solution method used during FEM analysis. Similar factors also affect the accuracy of FEM calculations in the area of the protrusible sleeve element itself. It seems that the only factor that affects the final result of the stiffness estimation, and is not included in the typical FEM calculations, is the compliance of the joint linking the prosthetic elements.

Although the modeling of contact problems is currently within the scope of functionality of various FEM software packages, this issue is difficult to solve in the case analyzed in this article, mainly due to insufficiently strictly defined contact conditions of protrusible sleeve and endoprosthesis body under the operating load. It seems that one of the methods of proceeding may be to introduce into the numerical calculations an additional intermediate element of small thickness, located between the cooperating elements of the joint, characterized by a certain degree of compliance. The mechanical characteristics of such element would be possible to be determined with the use of the results of experimental measurements. The procedure of experimental tests suggests that the stiffness of the joint can be determined e. g. by means of ESPI. The quantitative result of such a procedure could not be captured directly in a FEM model. A more complex method of numerical modeling taking into account the results of experimental tests proposed above may be a way to reduce a degree of inconsistency between FEM and ESPI results present in the current analysis and requires further research.

\section{Acknowledgments}

The work has been accomplished under the research project No. WZ/WM-IIB/2/2020. 


\section{REFERENCES}

1. Tschegg E.K., Herndler S., Weninger P., Jamek M., Stanzl-Tschegg S., Redl H. Stiffness analysis of tibia-implant system under cyclic loading. Materials Science and Engineering: C, 2008, 28, 1203-1208.

2. Buma F., Van Loon P.J.M., Versleyen H., Weinans H., Slooff T.J.J.H., De Groat E.C., Huiskes R. Histological and biomechanical analysis of bone and interface reactions around hydroxyapatite-coated intramedullary implants of different stiffness: a pilot study on the goat. Biomaterials, 1997, 18, 1251-1260.

3. Daas M., Dubois G., Bonnet A.S., Lipinski P., Rignon-Bret C. A complete finite element model of a mandibular implant-retained overdenture with two implants: Comparison between rigid and resilient attachment configurations. Medical Engineering \& Physics, 2008, 30, 218-225.

4. Hansson S., Ekestubbe A. Area moments of inertia as a measure of the mandible stiffness of the implant patient. Clinical Oral Implants Research, 2004, 15, 450-458.

5. Hohenhoff G., Haferkamp H., Ostendorf A., Meier O., Ostermeier S., Schimek M. Adapting Titanium Implants to the Elasticity of Bone by Comparison of Spring Stiffness. Advanced Engineering Materials. 2007;9(5):365-369.

6. Sumner D.R., Turner T.M., Igloria R., Urban R.M., Galante J.O. Functional adaptation and ingrowth of bone vary as a function of hip implant stiffness. Journal of Biomechanics, 1998, 31, 909-917.

7. Florin M., Arzdorf M., Linke B., Auer J.A. Assessment of stiffness and strength of 4 different implants available for equine fracture treatment: A study on a $20^{\circ}$ oblique long-bone fracture model using a bone substitute. Veterinary Surgery, 2005, 34, 231-238.

8. McCartney W., Mac Donald B.J., Hashmi M.S.J. Comparative performance of a flexible fixation implant to a rigid implant in static and repetitive incremental loading. Journal of Materials Processing Technology, 2005, 169, 476-484.

9. Roderer G., Gebhard F., Duerselen L., Ignatius A., Claes L. Delayed bone healing following high tibial osteotomy related to increased implant stiffness in locked plating. Injury, 2014, 45(10), 1648-52.

10. Schmidt U., Penzkofer R., Bachmaier S., Augat P. Implant Material and Design Alter Construct Stiffness in Distal Femur Locking Plate Fixation: A Pilot Study. Clinical Orthopaedics and Related Research, 2013, 471, 2808-2814.

11. Yavari S.A., Van der Stok J., Ahmadi S.M., Wauthle R., Schrootene J., Weinans H., Zadpoor A.A. Mechanical analysis of a rodent segmental bone defect model: The effects of internal fixation and implant stiffness on load transfer. Journal of Biomechanics, 2014, 47(11), 2700-2708.
12. Rohlmann A., Zander T., Bergmann G., Boustani H.N. Optimal stiffness of a pedicle-screw-based motion preservation implant for the lumbar spine. European Spine Journal. 2012, 21, 666-673.

13. Zander T., Rohlmann A., Burra N.K., Bergmann G. Effect of a posterior dynamic implant adjacent to a rigid spinal fixator. Clinical Biomechanics. 2006, 21, 767-774.

14. Rutherford S., Ness M.G. Effect of Contouring on Bending Structural Stiffness and Bending Strength of the 3.5 Titanium SOP Implant. Veterinary Surgery. 2012;41:983-987.

15. 15. Li X., Wang C.T., Zhang W.G., Li Y.C. Properties of a porous $\mathrm{Ti}-6 \mathrm{Al}-4 \mathrm{~V}$ implant with a low stiffness for biomedical application. Proceedings of IMechE Part H: Journal of Engineering in Medicine. 2009, 223, 173-178.

16. Foruria A.M., Carrascal M.T., Revilla C., Munuera L., Sanchez-Sotelo J. Proximal humerus fracture rotational stability after fixation using a locking plate or a fixed-angle locked nail: The role of implant stiffness. Clinical Biomechanics, 2010, 25, 307-311.

17. Kralinger F., Gschwentner M., Wambacher M., Smekal V., Haid C. Proximal humeral fractures: what is semi-rigid? Biomechanical properties of semi-rigid implants, a biomechanical cadaver based evaluation. Archives of Orthopaedic and Trauma Surgery, 2008, 128, 205-210.

18. Lill H., Hepp P., Korner J., Kassi J.P., Verheyden A.P., Josten C., Duda G.N. Proximal humeral fractures: how stiff should an implant be? A comparative mechanical study with new implants in human specimens. Archives of Orthopaedic and Trauma Surgery. 2003, 123, 74-81.

19. Borkowski P., Skalski K. Expandable endoprosthesis for growing patients - Reliability and research. Biocybernetics and Biomedical Engineering. 2014, 34, 199-205.

20. Koenigsberger F., Tlusty J. Machine Tool Structures. New York: Pergamon Press; 1970.

21. Mrozek P. The use of electronic speckle pattern interferometry for evaluation of machine tool static stiffness. Lasers in Engineering, 2019, 43(1-3), 81-99.

22. Yang L.X., Xie X., Zhu L.Q., Wu S.J., Wang Y.H. Review of electronic speckle pattern interferometry (ESPI) for three dimensional displacement measurement. Chinese Journal of Mechanical Engineering. 2014, 27, 1-12.

23. Będziński R., Tyndyk M. Experimental methods of stress and strain analysis in orthopaedics biomechanics. Acta of Bioengineering and Biomechanics. 2000, 2(2), 1-23.

24. Mrozek P., Mrozek E., Werner A. Electronic Speckle Pattern Interferometry for Vibrational Analysis of Cutting Tools. Acta Mechanica et Automatica. 2018, 12(2), 135-140. 